\title{
Guest editorial to the theme section on Multi-Paradigm Modeling for Cyber-Physical Systems
}

\author{
Eugene Syriani ${ }^{1} \cdot$ Manuel Wimmer ${ }^{2}$
}

Received: 16 March 2021 / Accepted: 17 March 2021 / Published online: 5 April 2021

(c) The Author(s), under exclusive licence to Springer-Verlag GmbH Germany, part of Springer Nature 2021

\begin{abstract}
This theme section aims to disseminate the latest research results in the area of Multi-Paradigm Modeling for Cyber-Physical Systems (MPM4CPS). MPM has a long tradition within the Model-Driven Engineering community, e.g., several workshops have been held at the MODELS conference for over more than a decade. The MPM4CPS workshop series is a continuation of the successful MPM workshop series with a stronger focus on CPS as especially these systems pose several new challenges on the engineering process and beyond. This theme section covers papers on the foundations and applications of MPM for CPS. In total, we accepted five submissions for publication in the theme section after a thorough peer-reviewing process.
\end{abstract}

Keywords Multi-paradigm modeling $\cdot$ Model-driven engineering $\cdot$ Systems engineering $\cdot$ Cyber-physical systems

\section{Introduction}

We are currently facing a dramatically increasing complexity in software and systems engineering with the emergence of cyber-physical systems (CPS) [2]. This increase of the complexity started already several decades ago when software components have been introduced as embedded systems for controlling physical parts. With CPS, this complexity is raised to a new level where the networking aspects of the systems and their (potentially open) environments are of paramount importance. In order to counteract this complexity, comprehensive and systematic views on all engineering disciplines including, e.g., mechanics, electronics, software, and network aspects, throughout the complete engineering life-cycle are a must. As an answer, model-based engineering

Eugene Syriani

syriani@iro.umontreal.ca

https://www.iro.umontreal.ca/ syriani/

Manuel Wimmer

manuel.wimmer@jku.at

https://www.se.jku.at/manuel-wimmer/

1 Department of Computer Science and Operations Research, University of Montreal, C.P. 6128, succ. Centre-Ville, Montreal, QC H3C 3J7, Canada

2 Christian Doppler Laboratory for Model-Integrated Smart Production (CDL-MINT), Institute of Business Informatics Software Engineering, Johannes Kepler University Linz, Altenberger Straße 69, 4040 Linz, Austria is emerging in industry to stay competitive and be successful in such settings. As such, model-based engineering has to be established on an enterprise-wide level as modeling is no longer considered to be limited to a single engineer, team, or discipline when developing CPS. However, this prominent use of models in engineering processes has raised several challenges which have to be tackled in order to ensure effective and efficient engineering processes for CPS. Nowadays, most of the complexity faced in CPS engineering is due to the huge number of cross-disciplinary design alternatives and complex inter-domain interactions, e.g., how to deal with continuous and discrete behaviors at the same time. Managing such inter-disciplinary engineering processes to ensure the development, delivery, deployment, operation, and evolution of CPS is required in all high-tech industries such as automotive, aerospace, railway, energy and manufacturing to mention just a few prominent examples.

Multi-paradigm modeling (MPM) offers the foundational framework for gluing the various engineering disciplines together in a consistent and systematic way [5]. The main principle of MPM is the use of explicit and heterogeneous models throughout the whole engineering life-cycle [5]. This leads to a framework where models are considered as firstclass citizens and are ubiquitous in the engineering processes. It has to be mentioned that models in this context can be used for many different purposes. For instance, some models represent the (visual) syntax of modeling formalisms. Other models are used to specify the transformations that represent 
the operational semantics of the modeling formalisms or they specify translations for inter-formalism transformation, e.g., for exchanging engineering models between different disciplines and tools. In addition, models may be used to represent the composition of other models, e.g., representing subsystems, or even the composition of modeling formalisms, e.g., for realizing co-simulation. On the operational level, models are used to facilitate language engineering tasks, e.g., evolving domain-specific modeling formalisms when engineering domains and their requirements are changing, but also tool engineering tasks are model-driven, e.g., for the automated generation of integrated (visual) modeling environments. The use of ubiquitous explicit models during the whole system engineering process, from modeling formalism definition to system realization, allows for various kinds of automated analyses at the most appropriate abstraction levels [5] with benefits in terms of performance, cost-effectiveness, safety, etc.

Multi-Paradigm Modeling for Cyber-Physical Systems (MPM4CPS) centers MPM on modeling problems encountered in systems which are composed of software and hardware components, even in some extent, including human actors [4]. The inherent complexity of CPS is managed by allowing different levels of abstraction and viewpoints, each expressed in the most appropriate modeling formalisms [4]. For this, MPM offers concepts, techniques, processes, and tools that allow to capture, combine, couple, and integrate each of the views that compose a system [5]. In this context, MPM covers a multitude of research topics: from engineering domain-specific modeling languages (including both, syntax and semantics), over multi-view and multi-abstraction modeling processes including simulation techniques for system analysis and optimization, to efficient and effective deployment of the systems. The added complexity of CPS compared to previous system generations, such as embedded systems and software-intensive systems, requires extensive research on how researchers and practitioners can apply or adapt existing MPM concepts, techniques, processes, and tools to this new application area, tying together multiple domains and engineering disciplines, and which new foundations are needed for MPM4CPS.

The Model-Driven Engineering (MDE) community has long embraced the need for MPM. Since the first workshop on MPM in 2006, a series of 10 workshops were co-located with the flagship conference in MDE: the International Conference on Model Driven Engineering Languages and Systems (MODELS). Many articles relying on MPM principles were also published in this journal, Software and Systems Modeling (SoSyM). The Computer Automated Multi-Paradigm Modelling (CAMPaM) [3] workshop has also gathered researchers and practitioners to identify and address grand challenges of MPM since 2004. Very recently, the MPM4CPS COST Action [1,4] running from 2014 to
2018 has led to numerous workshops and training schools and the establishment of a large inter-disciplinary research network concerned with the foundations and applications of MPM. In particular, two editions of the MPM4CPS workshop were co-located with MODELS in 2019 and 2020.

These initiatives have brought together researchers and practitioners in the area of MPM, specifically interested in developing CPS, to discuss synergies, common problems and solutions of the different engineering disciplines, tool building concerns and techniques, and very importantly, the vision for the future of the area.

\section{Selected papers for this theme section}

As the final outcome of a thorough peer review process, five papers were selected for this theme section:

- Moussa Amrani, Dominique Blouin, Robert Heinrich, Arend Rensink, Hans Vangheluwe, and Andreas Wortmann, in their paper "Multi-paradigm modelling for cyber-physical systems: a descriptive framework," present a unifying framework to describe modeling paradigms and their combinations. A particular focus is put on the concepts of formalism and workflow, which are in the center of a modeling paradigm. The proposed framework allows to capture the essence of a paradigm, to check whether a candidate modeling approach qualifies for a given paradigm, and to support the combinations of paradigms.

- Fatemeh Ghassemi, Iman Jahandideh, and Marjan Sirjani, in their paper "An actor-based framework for asynchronous event-based cyber-physical systems," present an extension of the language Timed Rebeca for modeling cyber-physical systems. The language extension is named Hybrid Rebeca and represents an actor-based language which allows to model physical actors to represent continuous behaviors. Hybrid automata can be derived from Hybrid Rebeca models for analysis purposes. The Hybrid Rebeca language is demonstrated in the automotive domain with the help of a case study. Results show that the analyzability and modularity of cyber-phyiscal systems can be improved with Hybrid Rebeca.

- Rodrigo Cortés Porto, Daniela Genius, and Ludovic Apvrille, in their paper "Handling causality and schedulability when designing and prototyping cyber-physical systems," present a dedicated model-driven framework for dealing with different models of computations during the design and verification phases of systems consisting of digital and analog parts. The focus is on statically detecting causality issues even before simulation or execution time. For this purpose, SysML models are employed and dedicated algorithms are presented. The 
approach is implemented in the TTool which also allows to derive virtual prototypes from SysML models.

- Sebastian Pilarski, Martin Staniszewski, Matthew Bryan, Frederic Villeneuve, and Dániel Varró, in their paper "Predictions-on-Chip: Model-based training and automated deployment of machine learning models at runtime," present a novel method to feed machine learning models for prediction with data coming from multiparadigm simulators. The resulting prediction models can be automatically deployed by using code generation techniques into control systems which have to deal with certain constraints such as programmable logic controllers. The method is evaluated in a real-world context covering three gas turbine bearings providing a huge number of data points for performing predictions.

- Adrien Le Coënt, Julien Alexandre dit Sandretto, and Alexandre Chapoutot, in their paper "Guaranteed master for interval-based cosimulation," present a co-simulation approach for continuous time systems. The core of the approach is a novel algorithm which reasons on global solutions by using only local computations. By this, a better scalability of the co-simulation approach can be reached with respect to existing approaches. The presented approach comes with tool support and is demonstrated on several examples.

Acknowledgements After more than a decade of running different events and initiatives concerning multi-paradigm modeling, there is a long list of people we like to thank. First, we are thankful to the authors of papers presented at the MPM4CPS workshops, and especially to those who submitted papers to this theme section. We also thank our reviewers for the timely manner in which they assisted in choosing and making suggestions to improve the selected papers in a especially challenging time period due to COVID-19. We would also like to warmly thank all co-organizers, PC members, and SC members of previous MPM4CPS workshops. Finally, we would like to express our gratitude to the SoSyM editorial office, specifically to Martin Schindler and Bernhard Rumpe who were always very helpful and supportive.

\section{References}

1. COST Action IC1404 Multi-Paradigm Modelling for CyberPhysical Systems. http://mpm4cps.eu/ (2014)

2. Lee, E.A.: Cyber physical systems: Design challenges. In: International Symposium on Object and Component-Oriented Real-Time
Distributed Computing, pp. 363-369. IEEE (2008). https://doi.org/ 10.1109/ISORC.2008.25

3. Mosterman, P.J., Vangheluwe, H.: Computer automated multiparadigm modeling: an introduction. Simulation 80(9), 433-450 (2004). https://doi.org/10.1177/0037549704050532

4. Vangheluwe, H., Amaral, V., Giese, H., Broenink, J.F., Schätz, B., Norta, A., Carreira, P., Lukovic, I., Mayerhofer, T., Wimmer, M., Vallecillo, A.: MPM4CPS: multi-paradigm modelling for cyberphysical systems. In: Joint Proceedings of the Doctoral Symposium and Projects Showcase Held as Part of STAF 2016, CEUR Workshop Proceedings, vol. 1675, pp. 40-47. CEUR-WS.org (2016)

5. Vangheluwe, H., De Lara, J., Mosterman, P.J.: An introduction to multi-paradigm modelling and simulation. In: AI, Simulation and Planning in High Autonomy Systems, pp. 9-20 (2002)

Publisher's Note Springer Nature remains neutral with regard to jurisdictional claims in published maps and institutional affiliations.

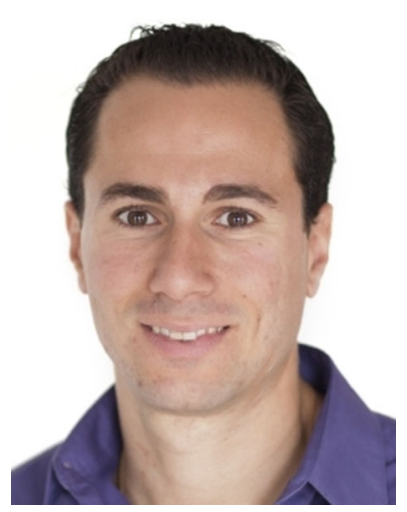

Eugene Syriani is an associate professor at the department of computer science and operations research at the University of Montreal. His main research interests fall in software design based on the model-driven engineering approach, the engineering of domain-specific languages, model transformation and code generation, simulation-based design, collaborative modeling, and user experience. For more information, please visit http://www.iro.umont real.ca/ $\sim$ syriani/.

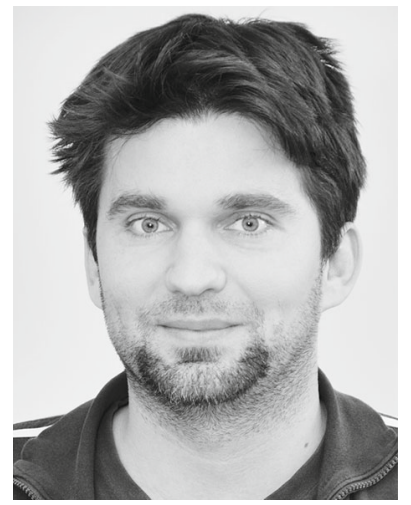

Manuel Wimmer is full professor leading the Institute of Business Informatics - Software Engineering at the Johannes Kepler University Linz and he is the head of the Christian Doppler Laboratory CDL-MINT. His research interests comprise foundations of model engineering techniques as well as their application in domains such as tool interoperability, legacy modeling tool modernization, model versioning and evolution, and industrial engineering. For more information, please visit https://www.se.jku.at/manuel-wimmer/. 